\title{
VARIABILITY OF SURFACE REFLECTION AMPLITUDES OF GPR HORN ANTENNA DEPENDING ON DISTANCE BETWEEN ANTENNA AND SURFACE
}

\author{
Jozef KOMAČKA ${ }^{1, *}$, Andrea PORUBIAKOVÁ 2 \\ ${ }^{1}$ Department of Highway Engineering, Faculty of Civil Engineering, University of Žilina, Univerzitná \\ 8215/1, 01026 Žilina, Slovakia. \\ 2 Department of Highway Engineering, Faculty of Civil Engineering, University of Žilina, Univerzitná \\ 8215/1, 01026 Žilina, Slovakia. \\ corresponding author: jozef.komacka@fstav.uniza.sk.
}

\begin{abstract}
The study focused on variability of surface reflections amplitudes of GPR horn antenna in relation to distance between an antenna and a surface is presented in the paper. The air-coupled antenna with the central frequency of $1 \mathrm{GHz}$ was used in the investigation. Four types of surfaces (dry pavement, wet pavement, metal plate and composite layer from gypsum and wood) were tested. The distance of antenna above the surfaces was changed in the range from $37.5 \mathrm{~cm}$ to 53.5 $\mathrm{cm}$. The amplitudes of negative and positive peaks and their variability were analysed in relation to the distance of antenna above the surfaces. Moreover, the influence of changes in the peaks of negative and positive amplitudes on the total amplitudes was assessed. It was found out the amplitudes of negative peaks for all investigated surfaces were relatively consistent in the range from 40.5 $\mathrm{cm}$ to $48.5 \mathrm{~cm}$ and the moderate decline was identified in the case of amplitudes of positive peaks in the range of distances from $37.5 \mathrm{~cm}$ to $51.5 \mathrm{~cm}$. This decline influences the tendency of total amplitudes. Based on the results of analysis it can be stated the distance of aircoupled antenna above the surface can influence the value of total amplitude and the differences depend on the type of surface.
\end{abstract}

Keywords:

Radar;

Pavement;

Surface;

Antenna;

Distance.

\section{Introduction}

Ground penetrating radar (GPR) is a system very often used in diagnostic of subsurface layers. Different types of antennas can be used depending on a required penetrating depth and resolution ability. Some types are in the contact with an investigated surface during diagnostic process (ground coupled antennas) others are in the recommended distance above the surface (air-coupled antennas). The latter type is very often used for road pavement diagnostic. Guideline regarding an optimum height of air-coupled antenna above a surface is obviously recommended by manufacturer of antenna. This is important for the scan resolution and the depth of penetration considering a possible reduction in radar performance. The different distance to surface could influence measured parameters and consequently also evaluated properties of pavement. A small study was performed to verify a variability of surface reflections amplitudes of GPR horn antenna in relation to distance between antenna and investigated surface.

\section{Experimental}

\subsection{Measurement and evaluation procedure}

Experimental measurements of amplitudes were performed with GPR system consisting of aircoupled antenna with the central frequency of $1 \mathrm{GHz}$, the control unit SIR-20 and the computer 
Toughbook. The antenna was placed above four types of surfaces: dry pavement, wet pavement, metal plate and composite layer consisting of gypsum plate with thickness of $13 \mathrm{~mm}$ and wooden plate with thickness of $39 \mathrm{~mm}$. The distance of the bottom side of antenna above the surface was changed and the used range covered the distance $(50 \mathrm{~cm}$ of little less) recommended in the manual of manufacturer of antenna [1].

The procedure of measurement (the same for all measurements) was as follows:

- Positioning of the antenna in the tested distance above the surface using support construction (vertical columns and horizontal pole),

- Stabilisation of signal for $15 \mathrm{~min}$,

- Running the measurements,

- Data recording for approx. $5 \mathrm{~s}$,

- Saving data.

The stored data were analysed in the software Radan 6.5 [2] using the O-scope mode (Fig. 1). The values of amplitudes, labelled in Fig. 1 as the Amp 1-u, Amp 1-b and Amp 1+, were read several times in the record of each measurement and minimum, maximum and average value was determined. Based on this, variability of amplitudes in relation to the distance between the antenna and investigated surface was assessed.

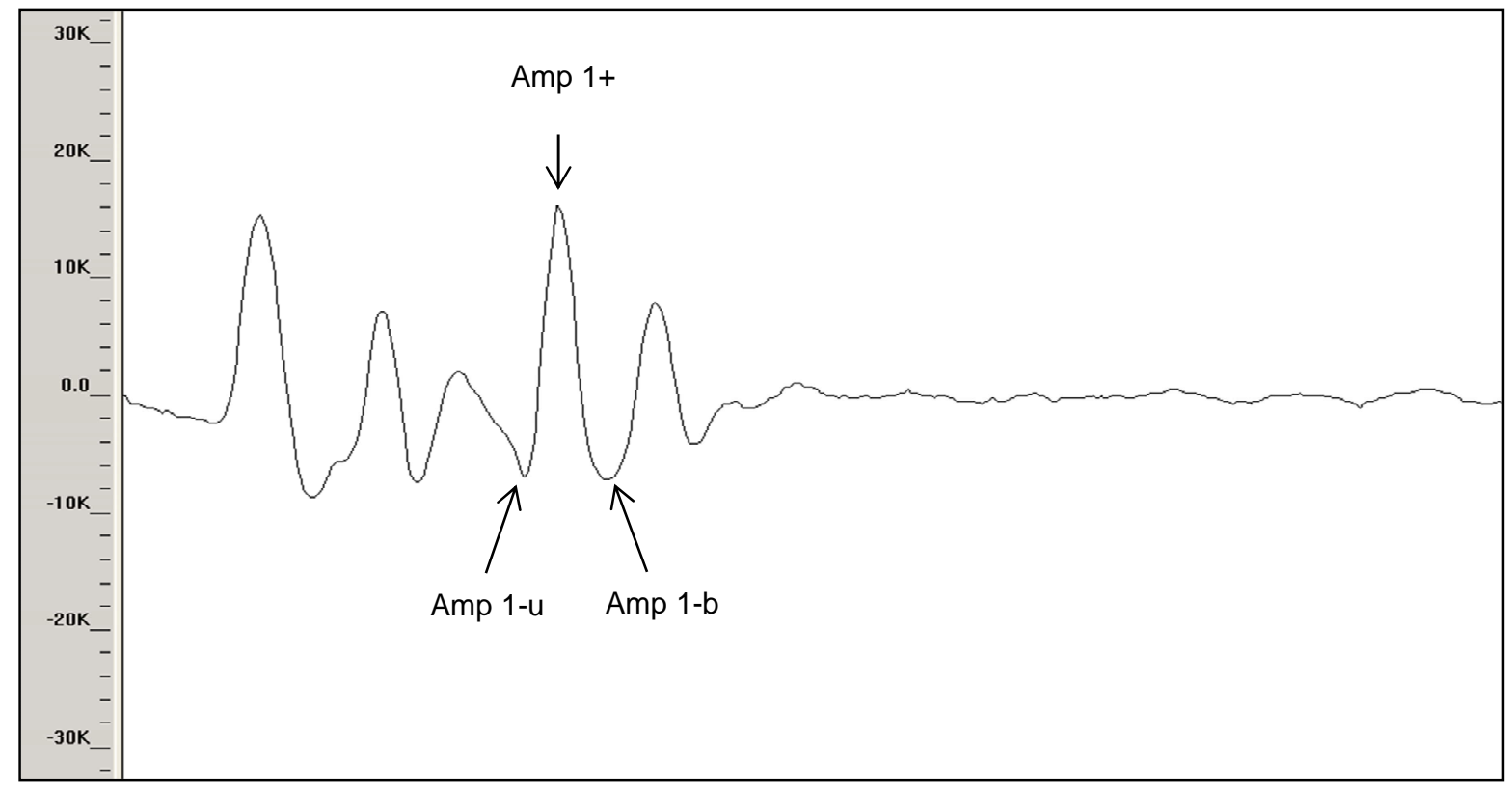

Fig. 1: Determination of amplitudes of surface reflection.

\subsection{Test results and discussion}

The values of amplitudes during a measurement were studied first. It was found out the amplitude is not the same despite there was no movement of antenna in vertical or horizontal direction. The example in Table 1 illustrates the amplitude for the same distance above the surface varies in some range. Moreover, the difference (Diff) between the minimum a maximum value is not constant for all distances between the antenna and investigated surface (Fig. 2). Interesting fact is there is not (actually in one case there was a very small) a difference for the amplitude Amp $1+$ in the case of metal plate regardless of distance. The numeric values of differences in Fig. 2 expressed in percentage of the minimum value are in the range from $4 \%$ up to $10 \%$. The exception is an amplitude Amp 1-b for the composite layer, where the difference is much higher due to low numeric value of amplitude. Based on the analysis mentioned above, the average values of amplitudes were used to assess the influence of distance between the bottom side of the antenna and the investigated surface. 
Table 1: Example of amplitudes variability measured on dry pavement surface.

\begin{tabular}{|c|c|c|c|c|c|c|c|c|}
\hline \multirow{2}{*}{$\begin{array}{c}\text { Distance } \\
\text { antenna-surface } \\
{[\mathbf{c m}]}\end{array}$} & \multicolumn{4}{|c|}{ Amp 1-u } & \multicolumn{4}{c|}{ Amp1+ } \\
\cline { 2 - 10 } & Min & Max & Diff & Avg & Min & Max & Diff & Avg \\
\hline 37.5 & -7045 & -6725 & 320 & -6842 & 15796 & 16436 & 640 & 16170 \\
\hline 40.5 & -5764 & -5124 & 640 & -5465 & 15049 & 15689 & 640 & 15412 \\
\hline 43 & -5231 & -4804 & 427 & -4996 & 14302 & 14942 & 640 & 14537 \\
\hline 46 & -5978 & -5444 & 534 & -5711 & 15156 & 15689 & 533 & 15380 \\
\hline 48.5 & -5978 & -5658 & 320 & -5732 & 14515 & 14942 & 427 & 14729 \\
\hline 51.5 & -5444 & -5231 & 213 & -5412 & 12274 & 13021 & 747 & 12615 \\
\hline 53.5 & -2669 & -2455 & 214 & -2605 & 5336 & 5763 & 427 & 5571 \\
\hline
\end{tabular}

Amp 1-u

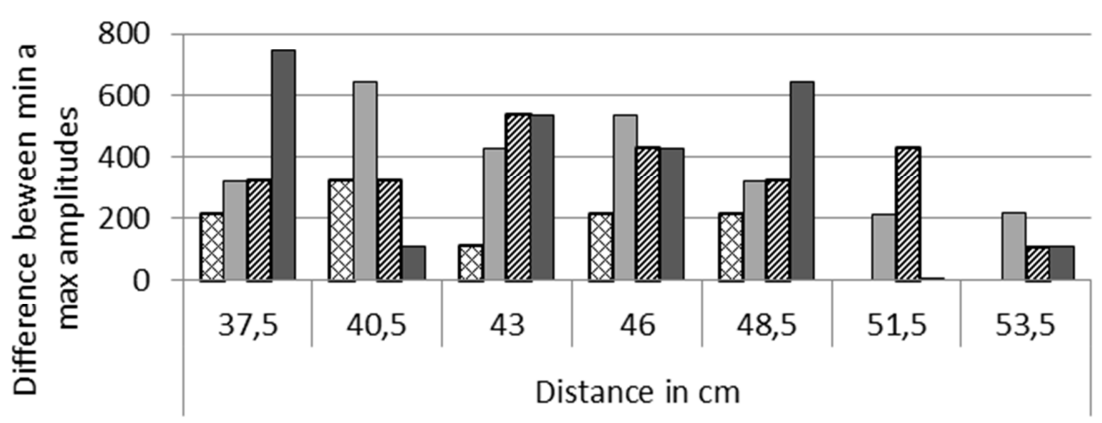

Amp 1+

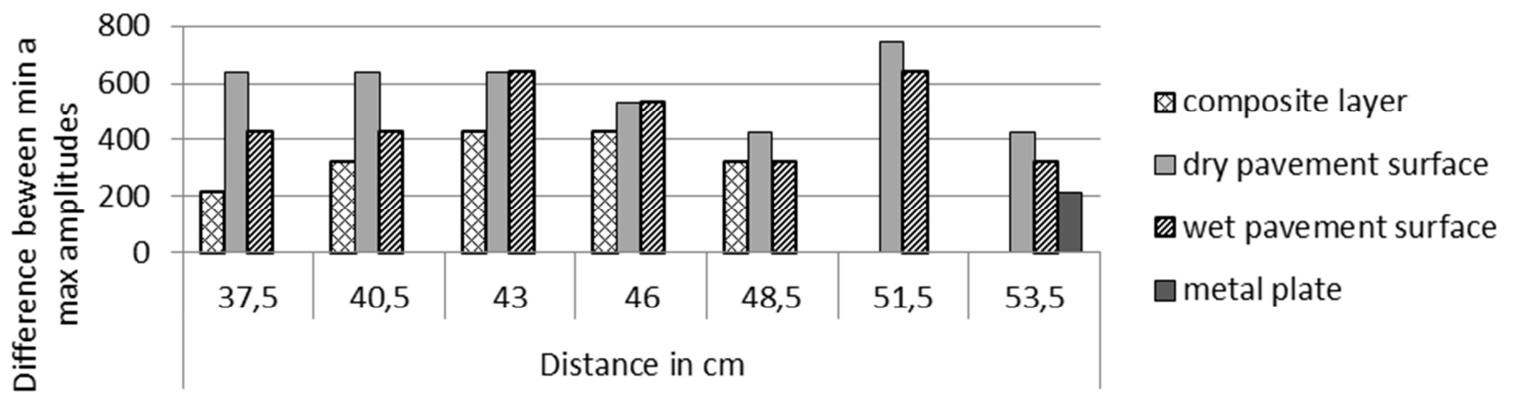

Amp 1-b

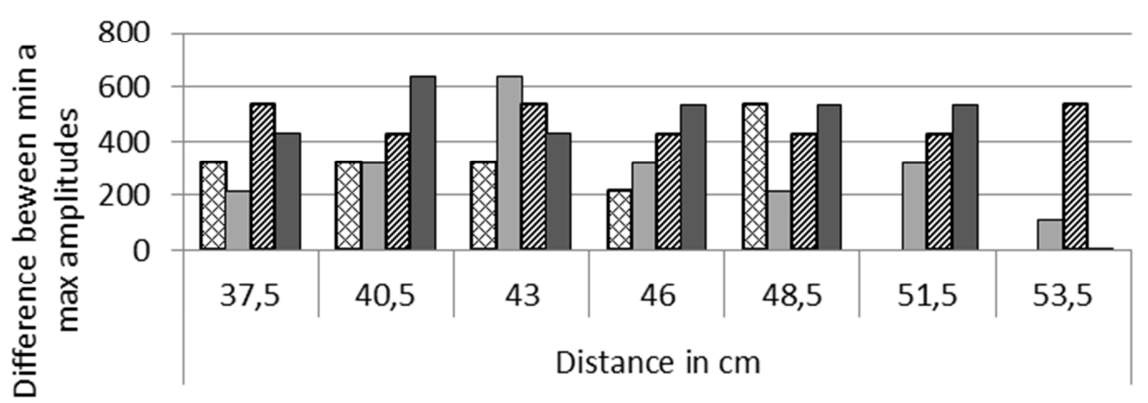

Fig. 2: Variability of differences between minimum and maximum value of amplitudes.

As it can be seen in Fig. 3, the average values of negative amplitudes for all investigated surfaces are relatively consistent in the range from $40.5 \mathrm{~cm}$ to $48.5 \mathrm{~cm}$. The differences exist, but taking into account the differences between the minimum and maximum value presented in Fig. 2 it could be stated the distance of the antenna above a surface has not any influence on the value of amplitude. Assessing the values of amplitudes at the marginal distance of $37.5 \mathrm{~cm}$ and the distance of $51.5 \mathrm{~cm}$, these amplitudes are slightly different when compared to the values in the interval from 
$40.5 \mathrm{~cm}$ to $48.5 \mathrm{~cm}$. The amplitudes at the distance of $53.5 \mathrm{~cm}$ (above the value recommended in [1]) are very different comparing to the others. Moreover, it is interesting, the values of amplitudes for the wet pavement surface are approximately equal (or in a narrow range) regardless of the distance of the antenna above the surface.

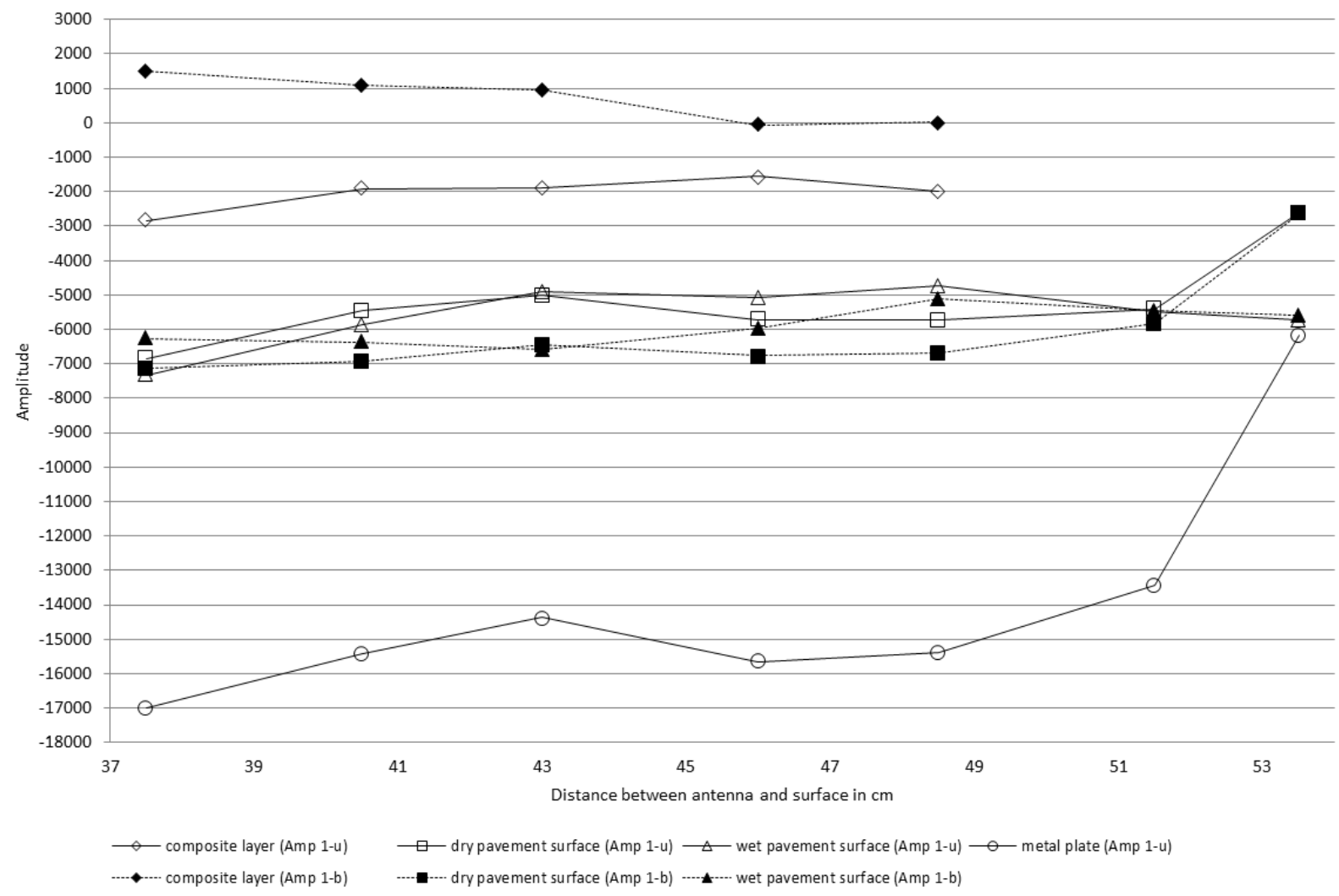

Fig. 3: Average amplitudes of negative reflections Amp 1-u and Amp 1-b.

Regarding positive amplitudes, the situation is slightly different. Except the amplitudes of reflection from the metal plate, a moderate decline can be identified for other types of surfaces in the range of distances from $37.5 \mathrm{~cm}$ to $51.5 \mathrm{~cm}$ (Fig. 4). The values at the marginal distance of 53.5 are much lower for the metal plate and the dry pavement surface. 


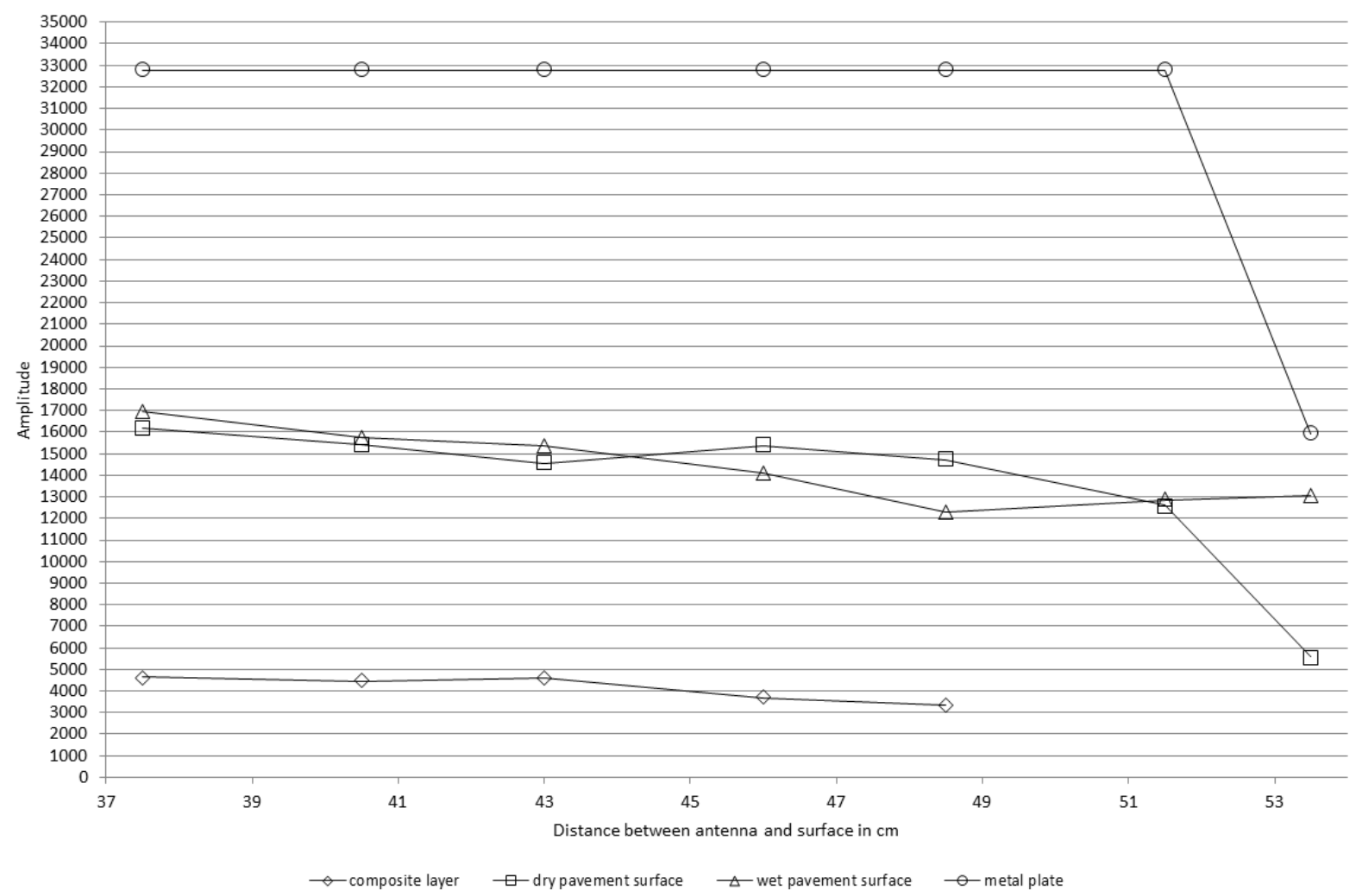

Fig. 4: Average amplitudes of positive reflections Amp 1+.

Taking into account the numeric values of positive amplitude, this decline can be important from the total amplitude (the sum of negative and positive amplitude) point of view. There are differences in total amplitude in Fig. 5 that demonstrate the deviation from the total amplitude for the distance of 48.5 $\mathrm{cm}$. It is clear that the difference of amplitudes increases with the distance from the reference distance of $48.5 \mathrm{~cm}$ and the course of numeric values of differences corresponds to the course of positive amplitudes. Only several are in the range that can be included in the range between the minimum and maximum value presented in Fig. 2. Others need to be assessed as data influenced by various distances of the antenna above surface.

These difference expressed in the percentage of reference value (i.e. the amplitude for the distance of $48.5 \mathrm{~cm}$ ) are shown in Fig. 6 . Only the values for the dry pavement surface and metal plate have small differences expressed as a percentage. The best situation is in the range between $40.5 \mathrm{~cm}$ and $48.5 \mathrm{~cm}$. Then, the deviation in percentage increases, mainly if the distance of the antenna above surface is higher than $50 \mathrm{~cm}$ or lower than $40 \mathrm{~cm}$. As for the composite layer or wet pavement surface, the differences in percentages reached very high values and increased with the distance of the antenna above surface. 


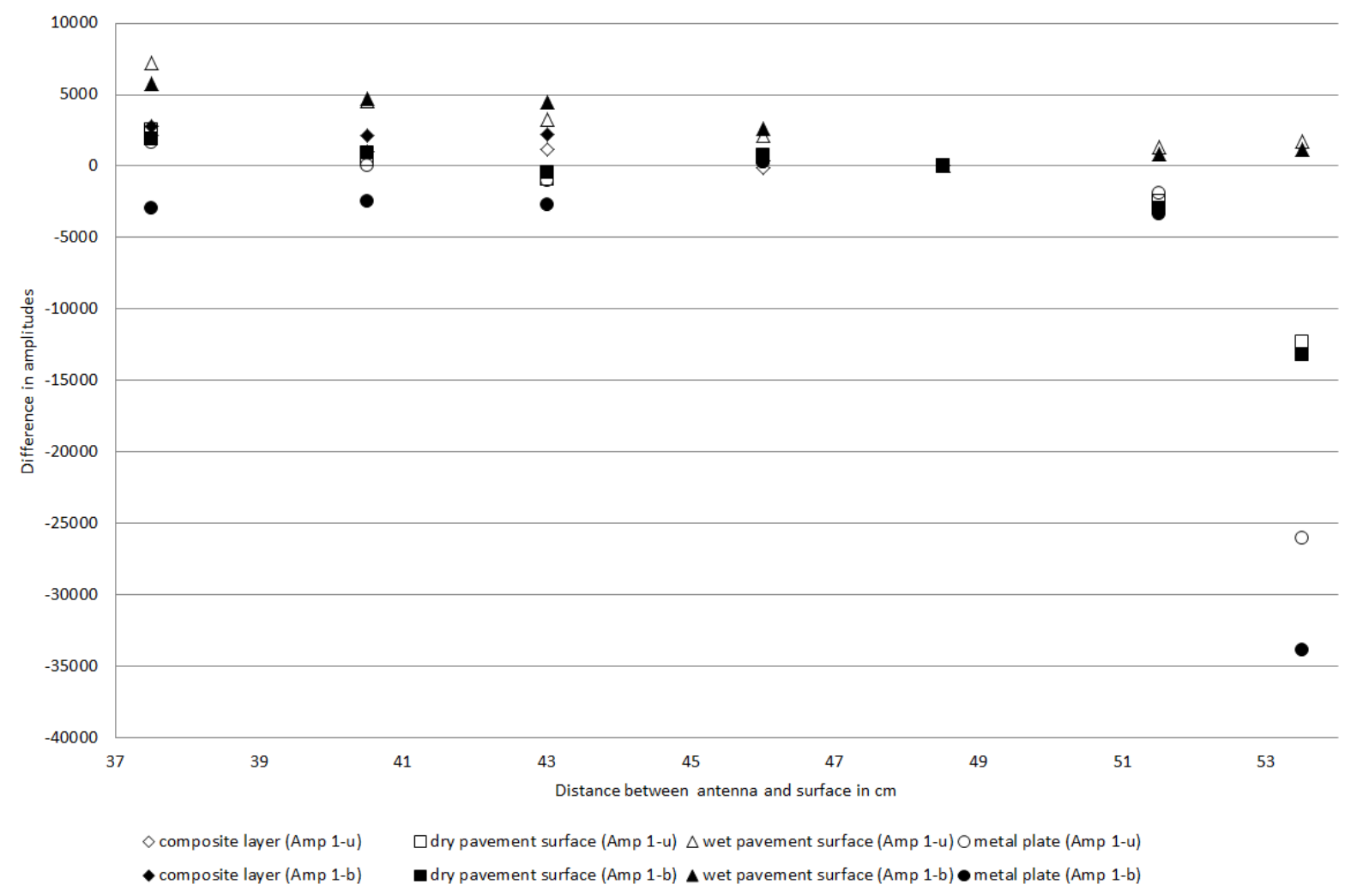

Fig. 5: Difference in amplitudes in relation to the reference value at the distance of $48.5 \mathrm{~cm}$.

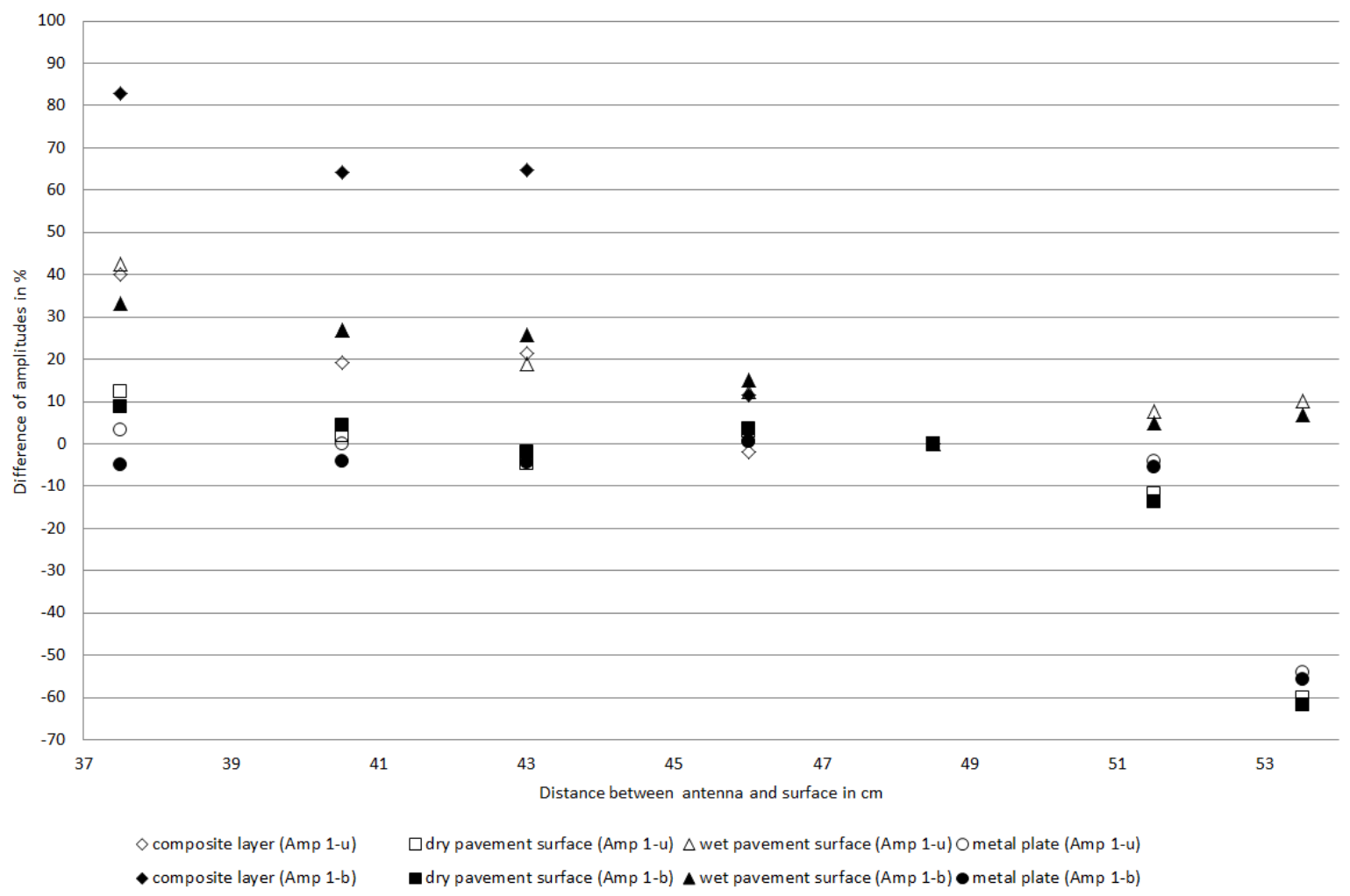

Fig. 6: Difference in amplitudes in the percentage of reference value. 


\section{Conclusions}

The results of measurements with the $1 \mathrm{GHz}$ air-coupled antenna have proven the amplitude of reflection for the same distance above a surface varies in some range. Moreover, the difference between the minimum a maximum value is not constant for all distances between the antenna and investigated surface. The numeric values of differences expressed in percentage of the minimum value of amplitude were in the range from $4 \%$ up to $10 \%$.

The amplitudes of negative peaks for all investigated surfaces were relatively consistent in the range from $40.5 \mathrm{~cm}$ to $48.5 \mathrm{~cm}$. The slightly deviations occurred at the distances of $37.5 \mathrm{~cm}$ and $51.5 \mathrm{~cm}$ and a significant deviation was found at the distance of $53.5 \mathrm{~cm}$.

The moderate decline was identified in the case of amplitudes of positive peaks in the range of distances from $37.5 \mathrm{~cm}$ to $51.5 \mathrm{~cm}$.

When the total amplitudes were analysed it was stated the decline of amplitudes of positive peaks influence the tendency of total amplitudes. The differences expressed in the percentage of the reference value (the total amplitude for the distance of $48.5 \mathrm{~cm}$ ) have shown slight deviations for the dry pavement surface and metal plate in the range of distances between $40.5 \mathrm{~cm}$ and $48.5 \mathrm{~cm}$. Unsatisfactory results were revealed when the distance of the antenna above surface was higher than $50 \mathrm{~cm}$ or lower than $40 \mathrm{~cm}$. Very high differences in percentages were identified for the composite layer and wet pavement surface and they increased with the distance of the antenna above surface.

Based on the results of analysis, it can be stated the distance of an air-coupled antenna above the surface can influence the value of total amplitude and the differences depend on the type of surface. This is an important finding since the total amplitude of surface reflection is used in some cases to determine the dielectric constant of the surface layer and the change in amplitude with distance could influence the calculated value of this parameter.

\section{Acknowledgement}

The paper is a result of research supported by research project VEGA 1/0557/14 Effect of selected variable parameters to asphalt pavement serviceability.

\section{References}

[1] Handbook for GPR inspections of road structures. MN43-183 Rev C. Geophysical Surveys Systems, Inc., 2007.

[2] Radan 6.5. User's manual. MN43-171 Rev D. Geophysical Surveys Systems, Inc., 2007. 